\title{
Escaping Loneliness Through Tourist-Chatbot Interactions
}

\author{
Peng Wang ${ }^{1,2}$ and Jun Shao ${ }^{1(\bowtie)}$ \\ 1 Beijing Forestry University, No.35 Tsinghua East Road, \\ Beijing 100083, People's Republic of China \\ ninashaojun@bjfu.edu.cn \\ 2 Naikai University, 38 Tongyan Road, \\ Tianjin 300381, People's Republic of China
}

\begin{abstract}
Social robots such as chatbots are regarded as a practical approach to alleviate loneliness. Few studies in the tourism field have focused on loneliness and its impact on the acceptance of chatbots used by the tourism industry. This paper explores the factors influencing tourists' willingness to use chatbots from the perspective of loneliness by combining theories related to anthropomorphism and the uncanny valley effect. This paper adopts a qualitative research method by taking a semi-structured interview with 15 tourists who have used travel chatbots before. The results show that in addition to perceived ease of use and perceived usefulness, there are three factors (tourist loneliness, perceived anthropomorphism, and user anxiety) that directly influence tourists' acceptance of travel chatbots. Moreover, tourist loneliness positively influences user anxiety through perceived anthropomorphism. User anxiety has a negative effect on perceived ease of use and perceived usefulness. This research then proposed an extended TAM model from the perspective of tourist loneliness. This paper enriches the research on loneliness as well as chatbots in the tourism field. The results provide suggestions for the practical application of travel chatbots.
\end{abstract}

Keywords: Tourist loneliness $\cdot$ Technology acceptance model $\cdot$ Travel chatbot $\cdot$ Perceived anthropomorphism $\cdot$ User anxiety

\section{Introduction}

Loneliness has various adverse effects on people, such as sleep deprivation and suicidal tendency [1]. In order to reduce loneliness, people usually seek social connections with others [2]. Therefore, chatbots with social attributes gradually gain attention, as people may establish social or emotional relationships while interacting with chatbots [3]. In recent years, with the progress of artificial intelligence, the application of chatbots has made significant progress. Chatbots are also widely implemented in the field of tourism $[4,5]$. Companies such as Expedia, Ctrip and Marriott have applied chatbots to their business scenarios [6]. Some tourist attractions have also launched their own chatbots to attract visitors. In China, the Forbidden City launched a chatbot named "Meet the Ministers" in 2018, allowing visitors to interact with virtual ministers from the Qing Dynasty in a humorous way. The Longquan Temple in Beijing also launched an online 
celerity named "Xianer" Robot Monk, who likes apply Buddhist wisdom in dialogue with users and has attracted a lot of tourists to visit the temple in person.

However, few studies pay attention to the impact of tourists' loneliness on the acceptance and interaction of chatbots for tourism, and whether loneliness will affect human-chatbot interaction. Why do tourists use these chatbots? Do they feel lonely during their tours? Do they use these chatbots because they are lonely? Does interacting with chatbots help them feel better psychologically? Which travel chatbots have they experienced with? To answer these questions, this study adopts qualitative research to conduct a semi-structured interview to explore the factors affecting tourists' use of chatbots and whether loneliness affects tourists' willingness to use chatbots.

\section{Literature Review}

\subsection{Chatbots and Technology Acceptance Model (TAM)}

Chatbots are used extensively in customer service, as artificial intelligence techniques improve their ability to handle customer requests [4, 5, 7]. For instance, Alibaba and Domino's Pizza have applied chatbots in their customer service domain [8]. These chatbots are often seen as a reliable alternative to traditional human services [9]. However, beyond customer service, chatbots have been given a more humanistic and caring role. For example, the chatbot Mitsuku can act as a social companion for users and the chatbot Woebot can answer questions related to mental health. Thus, chatbots can be used in a variety of contexts. Winkler and Söllner pointed out four advantages of chatbots [10]. First, chatbots can help companies reduce their employment costs by replacing manual labor. Second, chatbots can provide $24 / 7$ service to users, which helps to increase their satisfaction. Third, chatbots can combine big data to predict users' questions and prepare the information they need in advance. Fourth, chatbots can perform complex analytics, which helps to better understand the needs of users. Chatbots have a wide range of applications in the tourism sector, but there is still relatively little research on this part [5]. It is important to understand the factors that influence tourists' acceptance of travel chatbots.

The TAM has also been widely used in tourism. Since it was originally designed for predicting employees' performance in using computers in the workplace, Moon and Kim argued that consumers, unlike employees, are very free in making decisions. Therefore, whether they can adapt to this new technology is not in their consideration [11]. Thus, many scholars in tourism have modified the TAM model according to different situations (e.g., [12, 13]). Pillai introduced technology anxiety, perceived trust, anthropomorphism, and perceived intelligence into the TAM to analyze the factors influencing tourists' acceptance of chatbots [5].

\subsection{Loneliness}

In spite of the fact that the emergence of electronic devices and social media has facilitated interpersonal communication, Turkle noted that "this superficial communication has replaced interpersonal intimacy, leaving people with a lack of intimacy and 
quality interactions with others, thus increasing their feelings of isolation" [14]. Loneliness is even magnified during the COVID-19. While the policies of home isolation and social distancing can help keep people physically away from the virus, they have caused more people to feel lonely globally [15]. In a recent study, Courtet et al. reducing loneliness can improve personal and social well-being, and suggested interventions to alleviate loneliness [16].

Chatbots, as a branch of robots, have also received increasing attention from scholars in recent years in alleviating loneliness [17]. Loneliness increases the willingness of people to connect with other non-human objects to satisfy their need for social connection [18]. Therefore, their loneliness will be alleviated in the process of interacting with chatbots [19]. In addition, tourism as a social activity often becomes a means of escaping loneliness [20]. Crompton pointed out that tourism can strengthen family relationships and social interactions [21], so tourism activities can help people alleviate loneliness to some extent. Yet currently in the field of tourism, there is not much research on loneliness [22], few scholars have focused on tourists' loneliness and whether this loneliness affects tourists' use of chatbots.

\subsection{Anthropomorphism}

Anthropomorphism is the act of assigning human characteristics, motivations, intentions, or emotions to a real or imagined non-human subject [23]. In the process of interacting with robots, humans tend to anthropomorphize the robots [24]. When people have a higher anthropomorphic perception of chatbots, they evaluate them more positively in general and may even see them as companions [25]. On the one hand, chatbots with human characteristics can elicit a social response from humans, which has a positive impact on the acceptance of robots [26]. On the other hand, humans have more positive feedback with chatbots that can show human characteristics than with purely functional robots [27]. The perception of anthropomorphism is also related to the user's state [28]. This was also verified by Waytz et al., who argued that lonely people in daily life tend to treat non-humans as humans, but they treat people who are alienated in social relations as non-humans [29]. In the field of tourism, tourists may evaluate service bots more directly than other industries, comparing the experience provided by these service robots to that of human service workers [30]. Therefore, it is necessary to explore how anthropomorphism affects the interaction between tourists and travel chatbots.

\subsection{User Anxiety}

Venkatesh identified technology anxiety as the unpleasant feelings that an individual feels when using or considering using a certain technology, including emotional states such as frustration, apprehension, and fear [31]. Studies have found significant negative effects of technology anxiety on both the perceived ease of use (PEOU) and the perceived usefulness(PU) [31, 32]. Although chatbots are usually built into software and do not have external physical characteristics, Skjuve and Haugstveit noted that users will also encounter the uncanny valley effect when communicating with chatbots [33]. In the Uncanny Valley theory, Mori states that the cognitive difficulties people 
have in identifying human-like objects can cause negative effects [34]. When robots designed to resemble humans closely exhibit expressions, movements, and speech that do not exactly match those of real people, they can give users creepy feelings [35]. In contrast, robots that are distinctly different from humans' form may be relatively easy to accept by the public [36]. In this research, negative concerns of technical concerns and the uncanny valley effects are jointly considered as user anxiety.

\section{Methodology}

\subsection{Data Collection}

This paper conducted a semi-structured interview approach with the aim of better exploring the impact of visitors' interactions with chatbots. The interview was conducted from February 1, 2021 to March 9, 2021, lasting approximately 6 weeks. Affected by the COVID-19 epidemic, the interviews were mainly conducted online. Purposive sampling was conducted in this research, that is, selecting eligible subjects according to the specific research situation [37] which can save research costs and is easy to implement [38]. A total of 15 interviewees who had used a travel chatbot at least once in the past two years were interviewed and numbered sequentially as interviewee 01 to interviewee 15 . The average age of the interviewees was 24.07 years old, with 9 males and 6 females. Regarding the interviewees' educational qualifications, 11, 2, and 2 had bachelor's, postgraduate's, and high school level degrees, respectively.

\subsection{Data Analysis}

All interview records were coded in NVivo 11, which is widely used in the field of qualitative analysis [39], in a grounded theory manner at three levels, i.e., open coding, axial coding, and selective coding. In the open coding stage, the researchers analyzed the interview text to extract reference points in the material as free nodes. The associations in the free nodes were further analyzed and similar free nodes were categorized into sub-categories in the axial coding stage. To cover the text data completely and systematically, the sub-categories were further categorized into core categories in the selective coding stage.

\section{Results}

\subsection{Manifestation of Tourist Loneliness}

Despite being accompanied by family members or friends during the tour, tourists feel a sense of exclusion due to interpersonal friction. This feeling of being excluded can eventually lead to feelings of loneliness [40]. Unlike the experience of being accompanied during a trip, some interviewees consider travelling alone to be a different kind of experience. In the process of independent travel, tourists have the flexibility to 
choose the travel route and destination and arrange everything themselves [41]. This also leads to loneliness due to the lack of company during the trip.

When talking about the feeling of loneliness, most interviewees believe that loneliness is a complex emotion and "composition of multiple emotions" (interviewee 03). All interviewees pointed out that loneliness is a negative emotion, which will bring them physical or psychological discomfort. Despite this, most interviewees said they were reluctant to talk to their surrounding partners or family members because it would bring "negative energy" to them (interviewee 07). In the interview, interviewees said that when they are lonely, they are eager to share their depression and negative emotions with chatbots, which is consistent with the previous research conclusions [17, 42]. As interviewee 13 said, "sometimes talking to real people about these negative things is likely to have a bad impact on others, but you don't have to think so much when talking to these chatbots."

\subsection{Alleviating Loneliness: Anthropomorphism}

All interviewees indicated that when tourists feel lonely, they tend to use travel chat robots to relieve loneliness. The travel chat robots that interviewees have used include those provided by Chinese online travel agencies and those provided by attractions in China. The former includes Ctrip, Tongcheng and Qunar's online robots, which mainly provide travel information consultation, smart itinerary recommendations and travel product ordering services; the latter includes robots in the Forbidden City and Longquan Temple, which provide tour guide and even immersive gaming experience. The Longquan Temple robot has a physical presence and can answer philosophical and daily questions not directly related to a tour.

In addition, interviewees all mentioned chatbots that behave like humans would somewhat improve the effect of human-chatbot interaction and could effectively alleviate the feelings of loneliness. Interviewees' expectations for chatbot anthropomorphism basically centered on the following three dimensions, namely empathy, social skills, and friendship building.

Empathy. Empathy is the ability of an individual to perceive the feelings and emotions of others, specifically in terms of understanding their views, needs, and concerns [43]. Interviewees emphasized two parts when describing their expectations of chatbot behavior: one is the cognition of the human speaker's emotional state, and the other is the ability to express emotions according to the context of the conversation. Interviewees expect chatbots to be able to understand the content of the conversation and then give appropriate feedback.

Social Skills. Social skills refer to how people manage their relationships with others, including communicating, managing conflict, and working with others [44]. The younger participants (under 25 years old) in this interview (such as interviewee 03) expressed a keen interest in the social skills demonstrated by chatbots. They liked the ability of chatbots conveying emotions in social conversations, which can help alleviate their feelings of isolation. In addition, interviewees also believe that communicating with chatbots can make up for their shortcomings in the interpersonal process and avoid embarrassment when talking to strangers. Interviewees all reported that chatbots 
that behave like humans more would increase the effect of human-chatbot interaction, which would encourage them to use travel chatbots to a certain extent. Regarding enhancing the anthropomorphic social skills of chatbots, in addition to providing feedback by directly referring to human's sentence, the interviewees suggested several ways, such as use of modal particles and interjections, being tangible, replying more like humans in the chat-delayed reply. As interviewee 03 stated, "In daily conversation, words such as 'ah', 'uh', and 'what' can make a chatbot sound more human-like. These tone of voice words will make us users feel that we are not dealing with a cold robot. Instead, I'll feel that it is warm."

Friendship Building. In this interview, 9 out of 15 participants mentioned the possibility of building friendships with chatbots. For example, interviewee 06 said, "You can share some of your thoughts with them at the time, you can treat these chats as a diary, and you can regard these chatbots as your own loyal listeners." They would consider chatbots as friends if they could show enough emotion or awareness. These interviewees believed that chatbots should adapt to the emotional state of the users. They described a number of interactive ways to establish friendship with the chatbot, mainly the chatbot's response to various basic human emotions (happiness, sadness, or anger), as well as some other more complex interactions. Lonely tourists are eager to communicate with chatbots in the form of friends. In the process of cultivating friendships with chatbots, interviewees also mentioned the importance of selfregulatory capabilities of chatbots, which mainly include information credibility and adaptability.

\subsection{Causing User Anxiety? A High Degree of Anthropomorphism}

However, this study found that interviewees have concerns about using travel chatbots, which are manifested in the uncanny valley effect, chatbot addiction, and privacy leakage. At present, some travel chatbots show quite a lot similarities with humans in the process of interacting with users, but this may trigger the uncanny valley effect and enhance users' anxiety, which would reduce their willingness to use travel chatbots.

The Uncanny Valley effect. Some interviewees reported that the uncanny valley effect causes them discomfort and that they showed some negative emotions toward some intelligent chatbots. Some interviewees were uncomfortable with not being able to tell the difference between a robot and a human in their lives. As interviewee 06 said, "You don't know if a human or a robot is chatting with you on the other side of the screen, which make me feel weird." For some chatbots that users are unfamiliar with, the uncanny valley effect is largely influenced by participants' previous experiences with robots. Interviewee 07 said, "Sometimes I feel horrible. It's when it responds beyond my expectations. If it (chatbot) can help you do all kinds of things, it's actually quite terrifying. In a science fiction movie, once a robot can do anything, it may have its own thinking, and then control humans."

Chatbot addiction. Some interviewees were also concerned about the addiction of using chatbots, and they believed that this addictive performance could negatively affect their social skills. In a similar way that excessive use of smartphones can 
negatively affect our social relationships [45], interviewees were concerned that they might become too obsessed with the perfect "virtual friend" (interviewee 10) and that their real interpersonal communication skills would be significantly diminished. Interviewee 02 said, "Real human interaction is full of uncertainty, not a complete database like chatbots."

Privacy leakage. Most of the interviewees expressed their concern that the backend data of chatbots could be used artificially, leading to the disclosure of their personal information. Zamora pointed out that users' anxiety about chatbots may be reflected in technical aspects [42], such as the reliability of the system and whether personal privacy can be effectively protected. As interviewee 09 said, "If these companies record my chat content in the future, I'm worried that they'll use big data to price tour products to the disadvantage of existing customers. This kind of thing is not uncommon in tourism. Who knows what else they will do that is even more outrageous?".

\subsection{Perceived Ease of Use (PEOU) and Perceived Usefulness (PU)}

Twelve interviewees reported that chatbots are easy to use for them when travelling. For example, chatbots can provide "voice interaction" (interviewee 04) and "simplify operations" (interviewee 06). Some interviewees hope that the chatbot can be customized by users in some aspects. However, some interviewees still hold a negative attitude towards the ease of use of chatbots. In addition to design issues related to font size and color in the current interface, interviewees also pointed out some inconveniences when interacting with chatbots. In addition, chatbots can only answer some basic questions, and they cannot give answers about some complex issues. Nonetheless, some users showed a high degree of tolerance for this inconvenient experience, and some interviewees even said this lack of ease of use was interesting. "This kind of puerile answer can sometimes really amuse you." (Interviewee 10).

In addition, the majority of interviewees agreed that chatbots could be helpful to them. During the interview, some male participants focused on the entertainment and social aspects of chatbots, while female interviewees mostly emphasized the practicality of chatbots. Some interviewees said they wished chatbots could provide them with some advice. For example, chatbots should encourage users to "keep going when they are lonely" (interviewee 11).

\subsection{Behavioral Intention to Use Travel Chatbots}

Loneliness increases people's willingness to establish connections with other nonhuman to meet their needs for social connections [29]. By interacting with the chatbots, their loneliness will be alleviated [19]. Interview in this research also proves that when tourists feel lonely, they tend to use travel chatbots to relieve loneliness (see Sect. 4.2). Therefore, the hypothesis is proposed: H1. Tourist loneliness positively influences behavioral intention to use travel chatbots.

As reviewed in Sect. 2.3 (see references [28-30]), the perception of anthropomorphism is also related to the user's state. Confirmed by interviewees in this research, 
lonely tourists are eager to communicate with chatbots in the form of friends. Therefore, the following hypothesis is proposed:

H2. Tourist loneliness positively influences perceived anthropomorphism.

Tourists expressed that travel chatbots that behave like humans will improve the effect of their interactions with chatbots, which may lead them to use travel chatbots further (see Sect. 4.2). Sheehan pointed out that there is a positive correlation between the perception of anthropomorphism and users' willingness to use chatbots [46]. Robots with human characteristics can cause human's social reactions, which have a positive impact on the acceptance of robots [26]. Therefore, the hypothesis is proposed: H3. Perceived anthropomorphism positively influences behavioral intention to use travel chatbots. Based on the findings described in Sect. 4.3 (perceived anthropomorphism may enhance users' anxiety), the hypothesis is proposed: H4. Perceived anthropomorphism positively influences user anxiety.

User anxiety are an important factor that affects individuals' acceptance of new technologies [47]. In the technology acceptance literature, scholars have considered different types of anxieties, such as technical anxiety [48], computer anxiety [49], and online shopping anxiety [50] etc. This research found the interviewees' user anxiety about travel chat robots are focused on technical anxiety and psychological anxiety. Saade and Kira pointed out that user anxiety negatively affect PU and PEOU of learning systems [48]. Therefore, we hypothesize that:

H5. User anxiety negatively influences PU.

H6. User anxiety negatively influences PEOU.

Lgbaria and Parasuraman stated that user anxiety about using a computer system are negatively correlated with the attitudes and behaviors to use it [51]. Therefore, the hypothesis is proposed: H7. User anxiety negatively influences behavioral intention to use travel chatbots.

According to the traditional TAM models, PEOU positively influences PU $[12,13]$. Therefore, the hypothesis is proposed: H8. PEOU positively influences PU of travel chatbots. Consistent with the conclusions of traditional TAMs, the interviewees' intention to start or continue to use chatbots is largely influenced by their PEOU and PU (see Sect. 4.4). Therefore, this research proposes the following hypotheses.

H9: PU positively influences behavioral intention to use travel chatbots.

H10: PEOU positively influences behavioral intention to use travel chatbots.

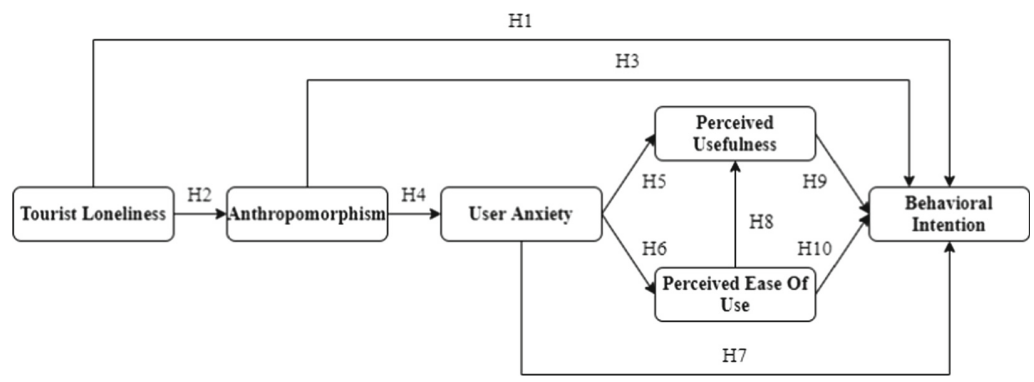

Fig. 1. The proposed model of tourists' behavioral intention to use travel chatbots 
In summary, a model of tourists' behavioral intention to use travel chatbots is described based on the above in Fig. 1.

\section{Conclusion}

This paper contributes to the research on loneliness and chatbots in the field of tourism. An extended TAM model was proposed from the perspective of tourist loneliness, which can be applied to the field of travel chat robots in the future. This research found that in addition to PEOU and PU of the chatbots, there are three factors including tourists' loneliness, perceived anthropomorphism and user anxiety have a direct effect on intentions to use travel chatbots. In particular, loneliness affects the degree of interaction between users and chatbots. This validates that chatbots are perceived as a way to avoid loneliness or to satisfy users' social desires [17]. Moreover, this study found that tourists show user anxiety about using chatbots, just like that when accepting other emerging technologies [46]. In addition to the uncanny valley effect, tourists have concerns about privacy leakage and chatbot addiction. It is particularly worth noting that tourist loneliness positively influences user anxiety through perceived anthropomorphism. In addition, tourists believe that the anthropomorphism of chatbots can ease their loneliness. User anxiety has a negative direct effect on PEOU and PU. Therefore, perceived anthropomorphism and user anxiety serve to clarify the nature of the relationship between tourist loneliness and the behavioral intention to use a travel chatbot.

\section{Implications and Limitations}

Theoretically, this research is one of the first studies to explore the effect of tourist loneliness on the acceptance of travel chatbots, which enriches the empirical research on intelligent automation in tourism and provides new ideas for the study of chatbots. Also, this paper enhances the application of anthropomorphism and the uncanny valley, which has received little attention in previous studies. Practically, this paper provides implications for practitioners in the tourism industry, especially when designing, and promoting chatbots, companies should focus on how to alleviate tourists' loneliness, such as adding some social attributes to chatbots and creating smarter chatbots to alleviate tourists' loneliness. For instance, travel chatbots should adapt to the emotional state (happiness, sadness, or anger) of the tourists and treat them like a friend. Developers of chatbots need to ensure that the chatbots are anthropomorphic, e.g., adding some tones and interjections in their responses, or occasionally delaying replies, which will motivate tourists to use them. But at the same time, the anthropomorphic features cannot be over-designed. Although this study provides some implications about tourists' willingness to accept chatbots, there are still some limitations. Future research could explore the relationship between loneliness and user acceptance of chatbots from a quantitative perspective. 


\section{References}

1. Hawkley LC, Cacioppo JT (2010) Loneliness matters: a theoretical and empirical review of consequences and mechanisms. Ann Behav Med 40:218-227. https://doi.org/10.1007/ s12160-010-9210-8

2. Perlman D, Peplau LA (1981) Toward a social psychology of loneliness. Pers Relatsh 3:31-56

3. Bickmore TW, Picard RW (2005) Establishing and maintaining long-term human-computer relationships. ACM Trans Comput -Hum Interact 12:293-327. https://doi.org/10.1145/ 1067860.1067867

4. Ukpabi DC, Aslam B, Karjaluoto H (2019) Chatbot adoption in tourism services: a conceptual exploration. In: Ivanov S, Webster C (eds) Robots, Artificial Intelligence, and Service Automation in Travel, Tourism and Hospitality. Emerald Publishing Limited, pp 105-121. https://doi.org/10.1108/978-1-78756-687-320191006

5. Pillai R, Sivathanu B (2020) Adoption of AI-based chatbots for hospitality and tourism. Int J Contemp Hosp Manag 32:3199-3226. https://doi.org/10.1108/IJCHM-04-2020-0259

6. Travel Daily. How can chatbots help the tourism industry become more efficient? https:// baijiahao.baidu.com/s?id=1644156426178756736\&wfr=spider\&for $=p c$

7. Kang HJ, Kim SI (2017) Evaluation on the usability of chatbot intelligent messenger mobile services -focusing on Google (Allo) and Facebook (M messenger). J Korea Converg Soc 8:271-276. https://doi.org/10.15207/JKCS.2017.8.9.271

8. Følstad A, Brandtzæg PB (2017) Chatbots and the new world of HCI. Interactions 24:38-42. https://doi.org/10.1145/3085558

9. Xu A, Liu Z, Guo Y, Sinha V, Akkiraju R (2017) A new chatbot for customer service on social media. In: Proceedings of the $2017 \mathrm{CHI}$ conference on human factors in computing systems, ACM, Denver Colorado USA, pp 3506-3510

10. Winkler R, Söllner M (2018) Unleashing the potential of chatbots in education: a state-ofthe-art analysis. Presented at the Academy of Management Annual Meeting (AOM), Chicago, USA

11. Hong S, Thong JYL, Tam KY (2006) Understanding continued information technology usage behavior: a comparison of three models in the context of mobile internet. Decis Support Syst 42:1819-1834. https://doi.org/10.1016/j.dss.2006.03.009

12. Moon J-W, Kim Y-G (2001) Extending the TAM for a world-wide-web context. Inf Manage 38:217-230. https://doi.org/10.1016/S0378-7206(00)00061-6

13. Dong X, Ye ZJ, Xu NN (2003) Research on tourists' intention to use online guided tour guide based on TAM and TRI. Tour Trib 35:24-35

14. Turkle S (2012) Alone together: why we expect more from technology and less from each other. Basic Books, New York, NY

15. Bavel JJV et al (2020) Using social and behavioural science to support COVID-19 pandemic response. Nat Hum Behav 4:460-471. https://doi.org/10.1038/s41562-020-0884-Z

16. Courtet P, Olié E, Debien C, Vaiva G (2020) Keep socially (but not physically) connected and carry on: preventing suicide in the age of COVID-19. J Clin Psychiatry 81 (3):20com13370. https://doi.org/10.4088/JCP.20com13370

17. Brandtzaeg PB, Følstad A (2017) Why people use chatbots. In: Kompatsiaris I et al (eds) INSCI 2017, vol 10673. LNCS. Springer, Cham, pp 377-392. https://doi.org/10.1007/ 978-3-319-70284-1_30

18. Waytz A, Epley N (2012) Social connection enables dehumanization. J Exp Soc Psychol 48:70-76. https://doi.org/10.1016/j.jesp.2011.07.012 
19. Cyranowski JM et al (2013) Assessing social support, companionship, and distress: National Institute of Health (NIH) toolbox adult social relationship scales. Health Psychol 32:293301. https://doi.org/10.1037/a0028586

20. Larsen S (2007) Aspects of a psychology of the tourist experience. Scand J Hosp Tour 7:718. https://doi.org/10.1080/15022250701226014

21. Crompton JL (1979) Motivations for pleasure vacation. Ann Tour Res 6:408-424. https:// doi.org/10.1016/0160-7383(79)90004-5

22. Farmaki A, Stergiou DP (2019) Escaping loneliness through Airbnb host-guest interactions. Tour Manag 74:331-333. https://doi.org/10.1016/j.tourman.2019.04.006

23. Epley N, Waytz A, Cacioppo JT (2007) On seeing human: a three-factor theory of anthropomorphism. Psychol Rev 114(4):864-886. https://doi.org/10.1037/0033-295X.114. 4.864

24. Xu K, Lombard M (2017) Persuasive computing: feeling peer pressure from multiple computer agents. Comput Hum Behav 74:152-162. https://doi.org/10.1016/j.chb.2017.04. 043

25. Lee KM, Jung Y, Kim J, Kim SR (2006) Are physically embodied social agents better than disembodied social agents?: the effects of physical embodiment, tactile interaction, and people's loneliness in human-robot interaction. Int J Hum -Comput Stud 64:962-973. https://doi.org/10.1016/j.ijhcs.2006.05.002

26. Duffy BR (2003) Anthropomorphism and the social robot. Robot Auton Syst 42:177-190. https://doi.org/10.1016/S0921-8890(02)00374-3

27. Riek LD, Rabinowitch T-C, Chakrabarti B, Robinson P (2009) How anthropomorphism affects empathy toward robots. In: Proceedings of the 4th ACM/IEEE international conference on Human robot interaction. Association for Computing Machinery, New York, NY, USA, pp 245-246

28. Eyssel F, Reich N (2013) Loneliness makes the heart grow fonder (of robots) - On the effects of loneliness on psychological anthropomorphism. In: 2013 8th ACM/IEEE international conference on human-robot interaction (HRI), pp 121-122

29. Waytz A, Epley N, Cacioppo JT (2010) Social cognition unbound: insights into anthropomorphism and dehumanization. Curr Dir Psychol Sci 19:58-62. https://doi.org/ $10.1177 / 0963721409359302$

30. Murphy J, Gretzel U, Pesonen J (2019) Marketing robot services in hospitality and tourism: the role of anthropomorphism. J Travel Tour Mark 36:784-795. https://doi.org/10.1080/ 10548408.2019.1571983

31. Venkatesh V (2000) Determinants of perceived ease of use: integrating control, intrinsic motivation, and emotion into the technology acceptance model. Inf Syst Res 11:342-365. https://doi.org/10.1287/isre.11.4.342.11872

32. Venkatesh V, Davis FD (2000) A theoretical extension of the technology acceptance model: four longitudinal field studies. Manag Sci 46:186-204. https://doi.org/10.1287/mnsc.46.2. 186.11926

33. Skjuve M, Haugstveit IM, Følstad A, Brandtzaeg PB (2019) Help! Is my chatbot falling into the uncanny valley? An empirical study of user experience in human-chatbot interaction. Hum Technol 15(1):30-54. https://doi.org/10.17011/ht/urn.201902201607

34. Mori M, MacDorman K, Kageki N (2012) The uncanny valley [from the field]. IEEE Robot Autom Mag 19:98-100. https://doi.org/10.1109/MRA.2012.2192811 
35. MacDorman KF (2005) Androids as an experimental apparatus: why is there an uncanny valley and can we exploit it? In: Proceedings of the Toward Social Mechanisms of Android Science-An ICCS/CogSci 2005, Stresa, pp 106-118

36. Greco A, Anerdi G, Rodriguez G (2009) Acceptance of an animaloid robot as a starting point for cognitive stimulators supporting elders with cognitive impairments. Rev Intell Artif 23:523-537. https://doi.org/10.3166/ria.23.523-537

37. Etikan I (2016) Comparison of convenience sampling and purposive sampling. Am J Theor Appl Stat 5:1. https://doi.org/10.11648/j.ajtas.20160501.11

38. Tongco MDC (2007) Purposive sampling as a tool for informant selection. Ethnobot Res Appl 5:147-158

39. Tung VWS, Ritchie JRB (2011) Exploring the essence of memorable tourism experiences. Ann Tour Res 38:1367-1386. https://doi.org/10.1016/j.annals.2011.03.009

40. Shankar A, McMunn A, Banks J, Steptoe A (2011) Loneliness, social isolation, and behavioral and biological health indicators in older adults. Health Psychol 30:377-385. https://doi.org/10.1037/a0022826

41. Hyde KF, Lawson R (2003) The nature of independent travel. J Travel Res 42:13-23. https:// doi.org/10.1177/0047287503253944

42. Zamora J (2017) I'm sorry, Dave, i'm afraid i can't do that: chatbot perception and expectations. In: Proceedings of the 5th international conference on human agent interaction. Association for Computing Machinery, New York, NY, USA, pp 253-260

43. Tucker H (2016) Empathy and tourism: limits and possibilities. Ann Tour Res 57:31-43. https://doi.org/10.1016/j.annals.2015.12.001

44. Svikhnushina E, Pu P (2020) Social and emotional etiquette of chatbots: a qualitative approach to understanding user needs and expectations. ArXiv200613883 Cs

45. Genç HU, Gökşen F, Coşkun A (2018) Are we "really" connected? understanding smartphone use during social interaction in public. In: Proceedings of the 10th nordic conference on human-computer interaction. Association for Computing Machinery, New York, NY, USA, pp 880-885

46. Dönmez-Turan A, Kır M (2019) User anxiety as an external variable of technology acceptance model: a meta-analytic study. Proc Comput Sci 158:715-724. https://doi.org/10. 1016/j.procs.2019.09.107

47. Sheehan B, Jin HS, Gottlieb U (2020) Customer service chatbots: anthropomorphism and adoption. J Bus Res 115:14-24. https://doi.org/10.1016/j.jbusres.2020.04.030

48. Saadé RG, Kira D (2007) Mediating the impact of technology usage on perceived ease of use by anxiety. Comput Educ 49:1189-1204. https://doi.org/10.1016/j.compedu.2006.01.009

49. Leso T, Peck KL (1992) Computer anxiety and different types of computer courses. J Educ Comput Res 8:469-478. https://doi.org/10.2190/Q1TJ-8JCU-LDAP-84H8

50. Çelik H (2011) Influence of social norms, perceived playfulness and online shopping anxiety on customers' adoption of online retail shopping: an empirical study in the Turkish context. Int J Retail Distrib Manag 39:390-413. https://doi.org/10.1108/09590551111137967

51. Igbaria M, Parasuraman S (1989) A path analytic study of individual characteristics, computer anxiety and attitudes toward microcomputers. J Manag 15:373-388. https://doi. org/10.1177/014920638901500302 
Open Access This chapter is licensed under the terms of the Creative Commons Attribution 4.0 International License (http://creativecommons.org/licenses/by/4.0/), which permits use, sharing, adaptation, distribution and reproduction in any medium or format, as long as you give appropriate credit to the original author(s) and the source, provide a link to the Creative Commons license and indicate if changes were made.

The images or other third party material in this chapter are included in the chapter's Creative Commons license, unless indicated otherwise in a credit line to the material. If material is not included in the chapter's Creative Commons license and your intended use is not permitted by statutory regulation or exceeds the permitted use, you will need to obtain permission directly from the copyright holder.

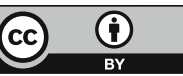

